\title{
Fatal Strongyloides stercoralis hyper-infection in a patient with multiple myeloma
}

Authors

Mohamed A Yassin, MD Halima El Omri, MD ${ }^{1}$ Ibrahim Al-Hijji, $\mathrm{MD}^{1}$ Ruba Taha, MD

Reham Hassan, MD

Kamal Al Aboudi, MD

Hanadi El-Ayoubi, MD ${ }^{1}$

${ }^{1}$ Hematology Department, Al Amal Hospital.
Submitted on: 06/02/2009 Approved on: 06/07/2009

Correspondence to: Dr. Mohamed A Yassin Al-Amal Hospital P.O.BOX 3050 Doha QATAR

Tel: +974 4397807 / +9744397895

Fax: +974 4397857

E mail:

yassin160@yahoo.co.uk

We declare no conflict of interest.

\begin{abstract}
Strongyloides stercoralis (S.S.) is a human intestinal parasite, which may lead to complicated strongyloidiasis. We report a case of disseminated strongyloidiasis following the treatment of myeloma. The patient developed skin lesions, respiratory distress, aseptic meningitis and bacterial and fungal sepsis. The diagnosis of strongyloidiasis was established through endotracheal tube secretions. Despite the treatment with Ivermectin and Albendazole, the outcome was fatal. The value of screening for strongyloidiasis is unclear but may be of benefit in patients with hematological malignancies from high endemic areas.
\end{abstract}

Keywords: acute respiratory failure, disseminated strongyloidiasis, myeloma, steroids.

[Braz J Infect Dis 2010;14(5):536-539]@Elsevier Editora Ltda.

\section{INTRODUCTION}

Strongyloides stercoralis (S.S.) is a nematode that infects approximately 100 million humans worldwide each year. S.S. is endemic in tropical and subtropical regions, such as Africa, Southeast Asia, and Southeastern United States, and occurs sporadically in temperate areas., ${ }^{1,2}$

The life cycle of S.S. in humans begins when free-living infective filariform larvae penetrate the skin and migrate hematogenously to the lung, ${ }^{1,3,4}$ where they penetrate into the alveolar air sacs. The larvae then ascend the tracheobronchial tree and are swallowed, thus accessing the duodenum and jejunum. The larvae develop into adult females, which lay eggs that hatch non-migratory (rhabditiform) larvae that penetrate the mucosae, leading to internal autoinfection. This autoinfection cycle may persist and dissemination has been reported to occur in immunocompromised hosts.

The risk factors for hyper-infection and disseminated disease are immunosuppressive therapy (particularly steroids), human T-lymphotropic virus 1 (HTLV1) infection, solid organ transplantation, hematologic malignant disease, especially lymphoma and hypoglob- ulinemia, hematopoietic bone marrow and stem cell transplantation, cancer chemotherapy, chronic alcohol consumption, uremia, severe malnutrition and diabetes mellitus. ${ }^{1,3-8}$ Of these, the most commonly reported risk factor is immunosupression due to steroids use. Out of more than 50 cases of hyperinfection and disseminated infection have been reported since 2000, the use of steroids was noted in $64 \%$ of cases. ${ }^{6}$ Steroids induce hyper-infection due to suppression of eosinophilia $^{3,9}$ and lymphocyte activation, and may also have a direct effect on the parasites themselves, accelerating the transformation of rhabditiform to invasive filariform larvae or rejuvenating reproductively latent adult females. ${ }^{1,3,6}$

Manifestations of infection can range from asymptomatic eosinophilia in the immunocompetent host to disseminated disease with septic shock in the immunocompromised host. Disseminated strongyloidiasis (D.S.) has been reported to occur in $1.5 \%-2.5 \%$ of all strongyloidiasis. In the absence of early diagnosis and treatment, the prognosis of (D.S.) is extremely poor. ${ }^{5}$

We present a case of a disseminated strongyloidiasis following the treatment of myeloma. 


\section{CASE REPORT}

The case was a 39-year-old Nepali man who had been resident in Qatar for the previous two years, not known to have any chronic disease. He was admitted to Hamad Medical Corporation on June 2007 with five days history of spinal cord compression symptoms. The investigation confirmed the diagnosis of IgD Lambda multiple myeloma with extramedullary localization, causing cord compression at different sites. The therapeutic plan was to treat him with the VAD protocol (Vincristine I.V. $0.4 \mathrm{mg} /$ day for four days, Doxurobicine $0.9 \mathrm{mg} / \mathrm{kg} /$ day for four days and Dexamethasone $40 \mathrm{mg} /$ day for four days [day 1 to day 4, day 9 to day 12, day 17 to day 21]), four to six cycles monthly followed by autologous hematopoietic stem cell transplant, in addition to radiotherapy for spinal cord compression. However, this protocol was delayed to avoid toxicity of concurrent chemoradiotherapy, so he was started only on high dose of Dexamethasone $40 \mathrm{mg}$ /day for four days, three boluses (day 1 to day 4, day 9 to day 12 , day 17 to day 21 ), and radiotherapy, 30 grays fractionated over 10 doses for two weeks on C3-D8. The patient also received $8 \mathrm{mg}$ of Dexamethasone between boluses to prevent inflammatory reaction secondary to radiotherapy; he was also kept on supportive measures (Cotrimoxazole $960 \mathrm{mg}$ P.O. twice per week, Omeprazole $20 \mathrm{mg}$ P.O. once daily, Zelodronic acid monthly and analgesics).

During the last session of radiotherapy he developed purpuric and serpiginous skin rash involving trunk, abdomen and upper thighs. Three days thereafter, he presented with bronchospasm, cough, hemoptysis, and dyspnea. The chest $\mathrm{x}$-ray showed bilateral pulmonary infiltrates suggesting severe infection or pulmonary embolism. The patient was then transferred to the medical intensive care unit (MICU) for progressive respiratory deterioration, where he had been intubated for assisted ventilation. During his stay in the MICU, he continued to bleed from the endothracheal tube (ETT), developed fever, and was treated empirically with Tazocin and Ciprofloxacin.

Figure 1: Wet mount from sputum.

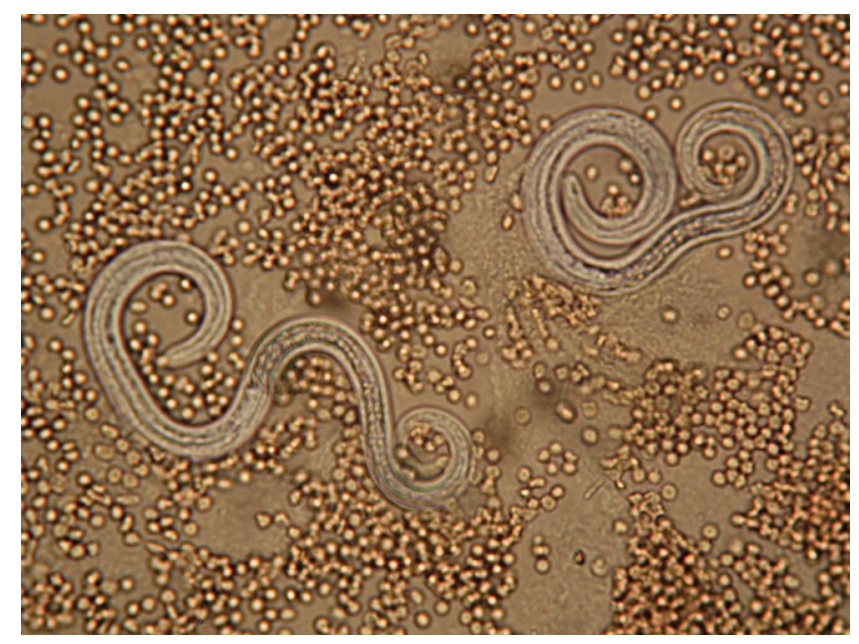

Figure 2: Wet mount from ETT.

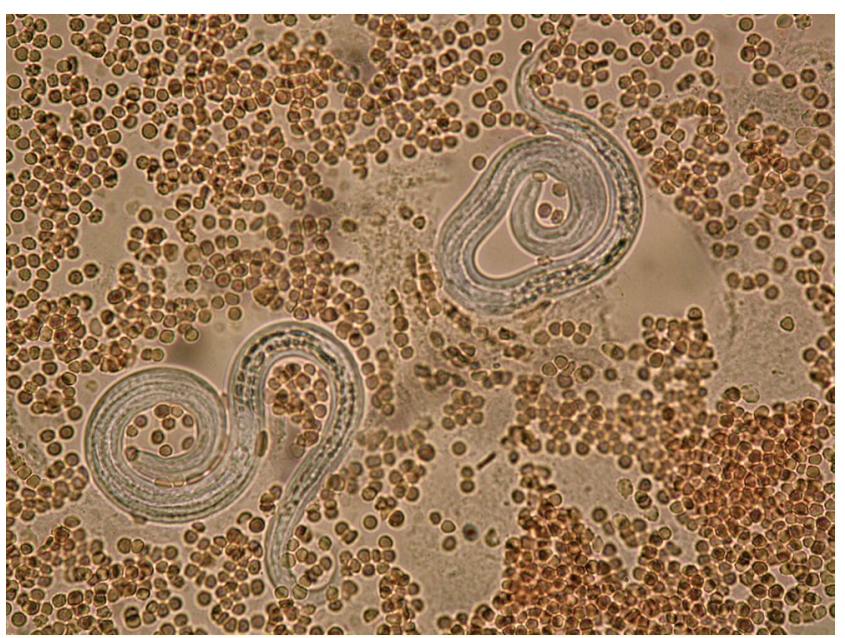




\section{Laboratory findings}

The laboratory findings were WBC $9.6 \times 10^{9} / \mathrm{L}$ with $90 \%$ neutrophils and $10 \%$ lymphocytes, $\mathrm{Hb} 13.5 \mathrm{~g} / \mathrm{dL}$, platelet $150 \times 10^{9} / \mathrm{L}$; Coagulation, liver function, and renal function were normal; Blood gas showed: $\mathrm{pH}$ 7.763, $\mathrm{PCO}_{2} 32 \mathrm{~mm} \mathrm{Hg}, \mathrm{Pa} \mathrm{O}_{2} 63 \mathrm{~mm} \mathrm{Hg}, \mathrm{HCO}_{3} 22 \mathrm{mmol} / \mathrm{L}$; Blood culture, urine culture and stool culture were negative, the endotracheal tube secretions revealed larvae of S.S. (Figures 1, 2 and 3) The skin biopsy turned out to be vasculitis, daily endothracheal tube (ETT) samples in the following two weeks revealed the same result (larvae of S.S.). Stool examination was repeatedly negative for S.S. Strongyloidiasis serology was not available. The patient was started on Albendazole $400 \mathrm{mg}$ daily through nasogastric tube for seven days and Ivermectin $200 \mathrm{mcg} /$ $\mathrm{kg}$ daily for two weeks.

Three weeks after his transfer to the MICU he had persistent fever, deterioration of the general conditions and developed skin herpetic lesions. Repeated ETT secretions revealed Pseudomonas aeruginosa, Candida albicans and hyphae filaments, but no larvae of S.S. Blood culture and stool cultures were negative, and urine culture showed Pseudomonas aeruginosa. Tazocin and Ciprofloxacin were switched to Meropenem and Vancomycin. Two days later Caspofungin and Acyclovir were added. In spite of antibiotic, antiparasitic, antifungal and antiviral treatment, the patient continued to have persistent fever, respiratory failure and developed neurological symptoms, like neck stiffness. Cerebrospinal fluid showed parameters suggestive of aseptic meningitis (WBC $130 / \mathrm{mm}^{3}$ with neutrophil $24 \%$, lymphocytes $76 \%$, protein $3.57 \mathrm{~g} / \mathrm{L}$, glucose $4 \mathrm{mmol} / \mathrm{L}$ and culture negative). Six weeks after his admission he died with septic shock.

Figure 3: Grain stain of sputum.

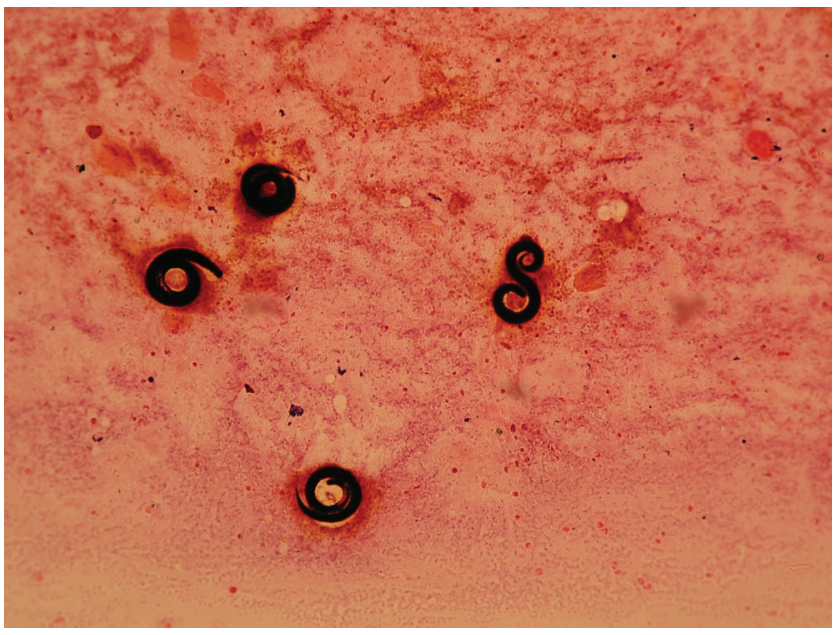

\section{DISCUSSION}

Strongyloidiasis has caused Strongyloides stercoralis (S.S.) infection. Manifestations of infection can range from asymptomatic eosinophilia in the immunocompetent host to disseminated disease in the immunocompromised host. ${ }^{3}$ The detection of increased number of larvae in the stool and/or sputum is a hallmark of hyper-infection.

In the case presented here, the patient was a citizen from $\mathrm{Ne}-$ pal, an endemic area for S.S. He did not have a past history of gastrointestinal and respiratory disorders. There was no eosinophilia in his peripheral blood at the time the myeloma was diagnosed. Our patient had intrinsic immunological abnormalities due to multiple myeloma (hypoglobulinemia) and received high dose of steroids and radiotherapy. These factors may have allowed the development of disseminated strongyloidiasis.

Clinically, hyper-infection syndrome or disseminated disease had seen reported to occur in up to $2.5 \%$ of all cases of strongyloidiasis infection. ${ }^{5}$ Most of the cases $(70 \%)$ occur in men, with a median age of 51 years. ${ }^{6}$ The clinical manifestation vary: fever, chills may not always be present, but mal-absorption syndrome, paralytic ileus, ulcerative enteritis and bleeding can complicate the disease. ${ }^{10}$

Pulmonary symptoms are among the most important signs of disseminated disease ranging from moderate to severe, including cough, wheezing, dyspnea, chest pain, hemoptysis, and a chocking sensation. In the absence of early diagnosis and treatment, the patient develops severe pulmonary hemorrhage and respiratory failure. The chest $\mathrm{x}$-ray most frequently shows bilateral focal interstitial infiltrates. ${ }^{5,11}$

Cutaneous symptoms are also frequent and include pruritus, urticarial serpiginous rash called larvae currens, which is pathognomonic of S.S infection. These lesions are usually located in the lower trunk and thighs. Petechial and purpuric rash of the same areas in which larvae have been demonstrated in skin biopsy are reported. Skin manifestations of vasculitis, the underlying disease and/or associated Gramnegative sepsis with disseminated intravascular coagulopathy may also be present during hyper-infection. ${ }^{3}$

Neurological manifestation can be seen: meningeal signs and symptoms is the most common indication of central nervous involvement. ${ }^{3,6}$ Usually, the spinal fluid show parameters of aseptic meningitis or demonstrates characteristics of Gramnegative bacterial infection; larvae can be found in spinal fluid, meningeal vessels, dural, epidural, subdural and subrachnoid spaces. However, it has been reported that all organs could be involved if the diagnosis is not made until later. ${ }^{1,3,5,6}$

Hyper-infection is often complicated by bacterial and fungal infection, ${ }^{1,3,6,11}$ because of the leakage of gut flora from a damaged bowel by moving larvae. Enteric bacteria are also carried by invasive larvae in their outer surfaces. This can result in septicemia, pneumonia, meningitis and disseminated bacterial or fungal infection in many parts of the body, ${ }^{1,11}$ massive secondary bacterial infections are 
frequently the immediate cause of death in patients with hyper-infection syndrome.

The clinical course of the present case is typical of strongyloidiasis. Initially, the patient developed purpuric and serpiginous skin rash involving trunk, abdomen, and upper thighs, and pulmonary symptoms including cough, bronchospasm, hemoptysis, and dyspnea. Previous infection with S.S. was not known and disseminated strongyloidiasis was not included in the differential diagnosis. Fortunately, identification of strongyloidiasis larvae in the ETT secretions confirmed the diagnosis. The patient also developed aseptic meningitis, bacterial and fungal pneumonia and urinary infection with Pseudomonas aeuruginosa.

The diagnosis in the immunocompromised (I.C) host is difficult because of a lower incidence of eosinophilia. The infection may be diagnosed by multiple stool samples. In chronic strongyloidiasis, examination of more than three stool samples has a sensitivity of $60 \%-70 \%$, and this may be higher in I.C. host. ${ }^{712}$ The separation and Bearman Concentration technique or stool culture may be used to improved detection of the larvae in stool. Infection may also be diagnosed by serology. A highly sensitive and specific Elisa serology has proven valuable in detecting both symptomatic and asymptomatic S.S. infection. ${ }^{1,6,12}$ Aspiration of duodenuo-jejunal fluid and small bowel biopsy or the use of strug test (Enterotest ${ }^{\circledR}$ ) may be required to detect S. larvae in such patients. ${ }^{10,12}$ Patients with pulmonary manifestations and suspected strongyloidiasis should have sputum culture and/or bronchoalveolar lavage ${ }^{5,11}$ for detection of S.S. in addition to ova, and parasites and Elisa testing.

In our case, the diagnosis was made later. The larvae of S.S. were revealed by ETT and the stool analysis was negative; the skin biopsy showed only vasculitis and serology was not done. Gastrointestinal endoscopy with aspiration of dudenuo-jejunal fluid and small bowel biopsy may have been required for earlier detection of SS. larvae, but the patient clinical condition did not allow further invasive procedures.

The goals of therapy for strongyloidiasis are to treat symptomatic disease and prevent complication in asymptomatic disease by eradicating the organism to prevent autoinfection, which could cause hyper-infection. ${ }^{12}$

For uncomplicated disease, current treatment options include Thiabendazole, Ivermectin and Albendazole. Thiabendazole $25 \mathrm{mg} / \mathrm{kg}$ twice daily for three days, or Albendazole $400 \mathrm{mg}$ daily for seven days, or Ivermectin $200 \mathrm{mcg} / \mathrm{kg}$ in one or two doses in patients weighting more than $15 \mathrm{~kg}$. The treatment is repeated if necessary 2-3 weeks after the first course to ensure eradication of infection. Follow up stool examination should be performed over a period of three months after treatment to confirm parasite eradication. ${ }^{6,13,14}$

For the hyper-infection syndrome, Ivermectin is recommended to be administered daily until symptoms resolve, and stool test have been negative for at least two weeks. ${ }^{6}$ Albendazole remains a potential alternative, but experience with its use is limited. ${ }^{4,14}$
Mortality rate due to hyper-infection could be as high as $87 \%{ }^{1}$ Most of the fatal infection caused by S.S. can be prevented by early detection and treatment of asymptomatic chronic infection.

Our patient was treated with Ivermectin $200 \mathrm{mcg} / \mathrm{kg}$ per day for two weeks and Albendazole $400 \mathrm{mg}$ daily for one week, but the outcome was unfavorable, because of late diagnosis at the stage of disseminated disease, which was complicated by bacterial and fungal infection.

The value of screening for strongyloidiasis is unclear, but may be of benefit in patients from high endemic areas, essentially before the start of corticotherapy and chemotherapy.

\section{ACKNOWLEDGEMENTS}

Thanks to Dr Doiphode Sanjay and Dr Deshmukh Anand from Microbiology Department Hamad Medical Corporation for their contribution to this article.

\section{REFERENCES}

1. Siddiqui AA, Berk SL. Diagnosis of Strongioloides stercoralis Infection. Clin Infect Dis 2001; 33:1040-7.

2. Genta RM. Global prevalence of strongyloidiasis: Critical review with epidemiologic insights into the prevention disseminated disease. Rev Infect Dis 1989; 11:755-67.

3. Keiser PB, Nutman TB. Strongyloides stercoralis in the immunocompromised population. Clin Microbiol Rev 2004; 17:208-17.

4. Hunter CJ, Petrosyan M, Asch M. Dissemination of Strongyloides stercoralis in a patient with systemic lupus erythematous after initiation of Albendazole: A case report. J Med Case Report 2008; 2:156-64.

5. Namisato S, Motomura K, Haranaga S et al. Pulmonary strongyloidiasis in a patient receiving prednisolone therapy. Inter Med 2004, 43:731-5.

6. Newnham MS. Manifestations, diagnosis, and treatment of Strongyloides stercoralis infection. Ann Pharmacother 2007; 41:1992-2001.

7. Orlent H, Crawley C, Cwynarski K, Dina R, Apperly J. Strongyloidiasis pre and post Autologous peripheral blood stem cell transplantation. Bone Marrow Transplant 2003; 32:115-7.

8. Carvalho EM, Dafonseca, Porto A. Epidemiological and clinical interaction Between HTLV-1 and Strongyloides stercoralis. Parasites Immunol 2004; 26:487-97.

9. Rotman HL, Yutanawiboonchai W, Brigande RA et al. Strongyloidess stercoralis: Eosinophil-dependant immune-mediated killing of third stage larvae in BALB/cByJ mice. Exp Parasitol. 1996; 82:267-78.

10. Concha R, Harrington W, Rogers AI. Intestinal strongyloidiasis recognition management, and determinant of outcomes. J Clin Gastrointerol 2005; 39:203-11.

11. Newberry AM, Williams DN, Stauffer WM, et al. Strongyloides hyperinfection presenting as acute respiratory failure and gramnegative sepsis. Chest 2005; 128:3681-4.

12. Lim S, Katz K, Kratjden S, et al. Complicated and fatal strongyloides infection in Canadian: risk factors, diagnosis and management. CMAJ 2004; 171:479-84.

13. Igual-Adell R, Oltra-Alcaraz C, Soler-Company E, et al. Efficacy and safety of Ivermectin and Thiabendazole in the treatment of strongyloidiasis. Expert Opinion Pharmacother 2004; 5:2615-9.

14. Muennig P, Pallin D, Challah C, et al. The cost effectiness of Ivermectin vs. Albendazole in the presumptive treatment of strongyloidiasis in immigrants to the United States. Epidemiol Infect 2004; 132:1055-63. 